\title{
XNAT imaging platform for BioMedBridges and CTMM TralT
}

Stefan Klein ${ }^{1 *}$, Erwin Vast ${ }^{1}$, Johan van Soest ${ }^{2}$, Andre Dekker $^{2}$, Marcel Koek ${ }^{1}$, Wiro Niessen ${ }^{1,3}$

From 1st Clinical Research Informatics (CRI) Solutions Day

Duesseldorf, Germany. 26-27 May 2014

\section{Characterisation}

Service, imaging, open source, archive, image sharing, clinical trials.

\section{Description}

The XNAT imaging platform is a web service for storing, organizing, and sharing medical imaging data. It is based

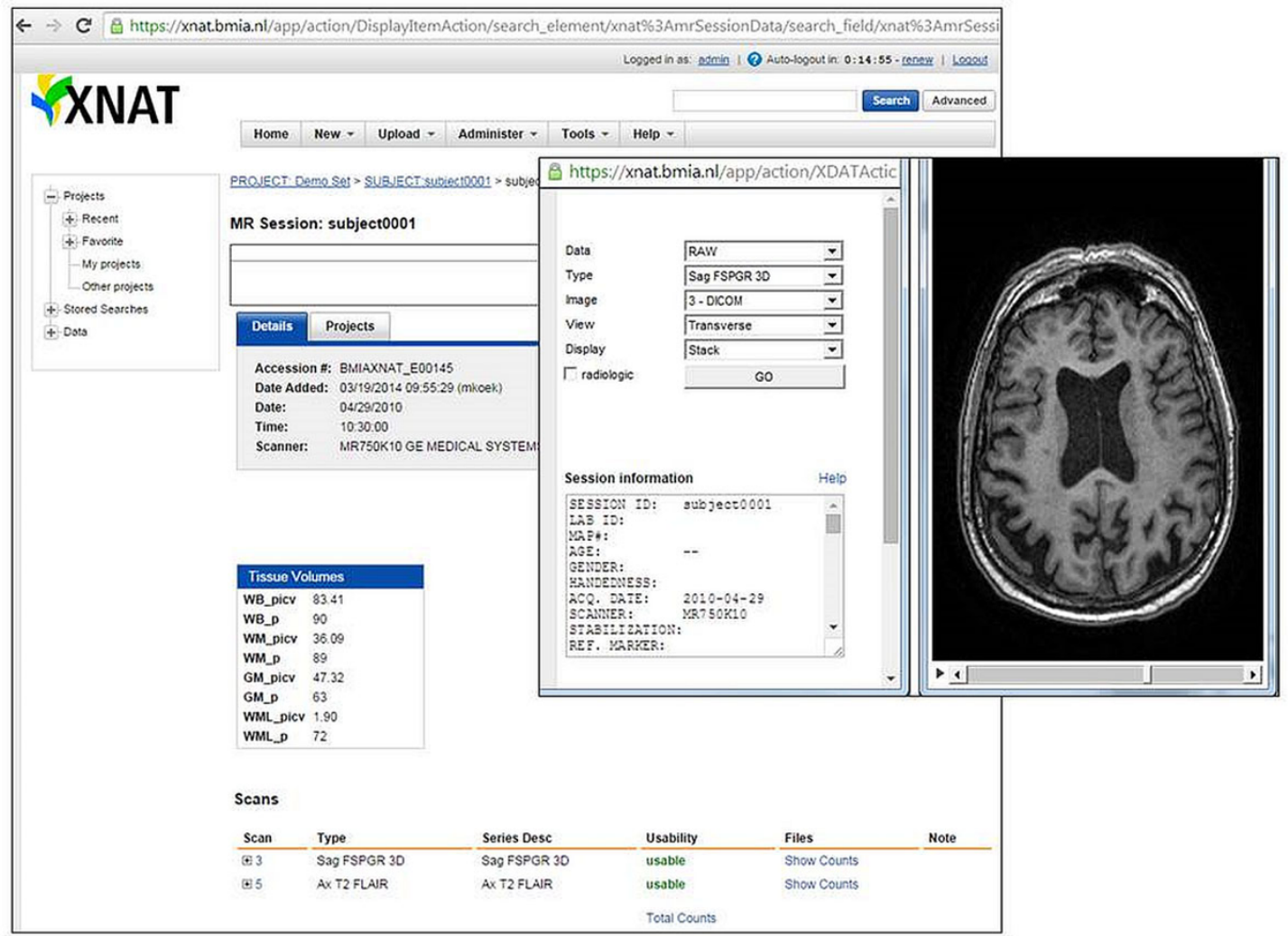

Figure 1 XNAT user interface. Example project with magnetic resonance imaging (MRI) data of the brain.

\footnotetext{
* Correspondence: s.klein@erasmusmc.nl

'Biomedical Imaging Group Rotterdam, Departments of Medical Informatics

\& Radiology, Erasmus MC, 3000CA Rotterdam, the Netherlands

Full list of author information is available at the end of the article
} 
on the open source eXtensible Neuroimaging Archive Toolkit (XNAT, http://www.xnat.org) [1]. The platform is suitable for both single-center and multi-center clinical studies with imaging data (Figure 1). Medical imaging data (in DICOM format) and image-derived data (often in non-DICOM format) are supported. Downloading and uploading is possible via the web interface, via the DICOM protocol, and via a RESTful application programming interface. Read and write access rights can be controlled per project and per user. In the context of the European BioMedBridges project and the Dutch CTMM TraIT project, two XNAT instances have been put in production. In the CTMM TraIT instance, which is especially supports multi-center clinical imaging studies, submission of DICOM imaging data is performed via the RSNA Clinical Trial Processor (CTP) to enforce proper anonymisation. In order to simplify and automate the installation of XNAT, we developed scripts based on the "Puppet" configuration software. These scripts have been made publicly available via the XNAT marketplace (http://marketplace.xnat.org).

\section{Status of development}

A recent stable release of the XNAT software is used. Currently (since November 2014), we are running version 1.6.2.1. The XNAT instances are in use by several research projects. The configuration scripts are in active development, and the current release is version 0.2 alpha.

\section{Users}

Researchers using medical imaging data.

\section{Links}

Documentation and links to both XNAT instances and Puppet installation scripts [http://xnat.bigr.nl]; documentation of RSNA CTP [http://mircwiki.rsna.org/ index.php?title=CTP_Articles].

\section{Acknowledgments}

The BioMedBridges project is funded by the European Commission within Research Infrastructures of the FP7 Capacities Specific Programme, grant agreement number 284209. The TralT project is an initiative from the Center for Translational Molecular Medicine (CTMM).

\section{Authors' details}

${ }^{1}$ Biomedical Imaging Group Rotterdam, Departments of Medical Informatics \& Radiology, Erasmus MC, 3000CA Rotterdam, the Netherlands. ${ }^{2}$ Maastricht UMC+, MAASTRO Clinic, Maastricht, the Netherlands. ${ }^{3}$ Imaging Physics, Applied Sciences, Delft University of Technology, the Netherlands.

Published: 22 May 2015

\section{Reference}

1. Marcus DS, Olsen T, Ramaratnam M, Buckner RL: The Extensible Neuroimaging Archive Toolkit (XNAT): An informatics platform for managing, exploring, and sharing neuroimaging data. Neuroinformatics 2007, 5(1):11-34.

doi:10.1186/2043-9113-5-S1-S18

Cite this article as: Klein et al: XNAT imaging platform for

BioMedBridges and CTMM TralT. Journal of Clinical Bioinformatics 20155 (Suppl 1):S18.

\section{Submit your next manuscript to BioMed Central} and take full advantage of:

- Convenient online submission

- Thorough peer review

- No space constraints or color figure charges

- Immediate publication on acceptance

- Inclusion in PubMed, CAS, Scopus and Google Scholar

- Research which is freely available for redistribution

Submit your manuscript at www.biomedcentral.com/submit 